\title{
Total solidification time of a liquid phase change material enclosed in cylindrical/spherical containers
}

\author{
Levent Bilir *, Zafer İlken \\ Department of Mechanical Engineering, İzmir Institute of Technology, Gülbahçe-Urla, 35430, İzmir, Turkey \\ Received 14 April 2004; accepted 8 October 2004 \\ Available online 16 December 2004
}

\begin{abstract}
This study investigates the inward solidification problem of a phase change material (PCM) encapsulated in a cylindrical/spherical container with a third kind of boundary condition. The governing dimensionless equations of the problem and boundary conditions are formulated and solved numerically by using enthalpy method with control volume approach. The problem is solved many times for different values of the affecting parameters and data sets are obtained for dimensionless total solidification time of the PCM. These data sets are then used to derive correlations which express the dimensionless total solidification time of the PCM in terms of Stefan Number, Biot Number and Superheat Parameter.
\end{abstract}

(C) 2004 Elsevier Ltd. All rights reserved.

Keywords: Phase change; Latent heat energy storage; Inward solidification in cylindrical and spherical region

\section{Introduction}

Cool storage systems remove heat from a thermal storage medium during the periods of low cooling demand and use this cool energy when it is needed. For that purpose, latent heat systems are more attractive than sensible ones due to their large storage capacities and constant charge and discharge temperatures. One of most popular latent heat storage systems is the encapsulated

\footnotetext{
${ }^{*}$ Corresponding author. Tel.: +90 232 7506605; fax: +90 2327506505.

E-mail address: leventbilir@iyte.edu.tr (L. Bilir).
} 


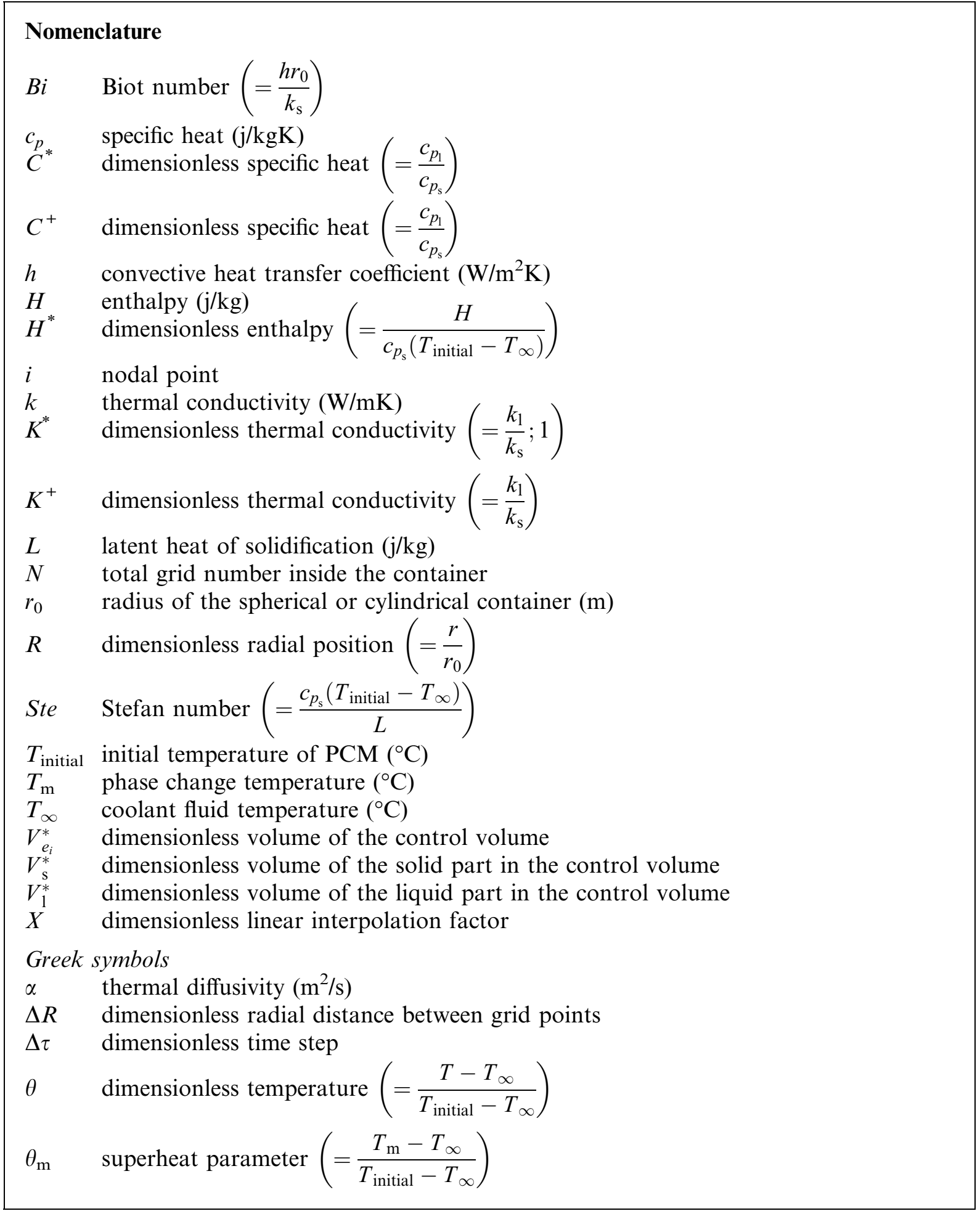




\begin{tabular}{ll|}
$\tau$ & dimensionless time $\left(=\frac{\alpha_{\mathrm{s}} t}{r_{0}^{2}}\right)$ \\
$\tau_{\text {total }}$ & dimensionless total solidification time \\
Subscripts & control volume face between $P$ and $E$ \\
$\mathrm{E}$ & neighbour grid point on the east side \\
$\mathrm{l}$ & liquid phase \\
$\mathrm{P}$ & central grid point under consideration \\
$\mathrm{S}$ & solid phase, Interface \\
$\mathrm{W}$ & control volume face between $P$ and $W$ \\
$\mathrm{~W}$ & neighbour grid point on the west side \\
& \\
Superscript \\
$k$ & time level
\end{tabular}

PCM systems, which uses cylindrical or spherical containers packed into a storage tank. These geometries are commonly preferred due to their favourable proportion of the energy storage volume to the heat transfer surface area.

Tao [1] presented a numerical method for the problem of freezing a saturated liquid inside a cylindrical or a spherical container. The heat capacity and thermal conductivity of solid phase and the heat transfer coefficient are assumed to be constants. Cho and Sunderland [2] obtained an approximate solution for the inward and outward phase change of a spherical body. The initial temperature of the body is assumed constant at the fusion value and the surface temperature is assumed to change instantaneously. The accuracy of the approximation is checked by a finite difference formulation. Shih and Tsay [3] generated successive approximate solutions for the freezing of a saturated liquid inside a cylindrical container under the constant heat transfer coefficient boundary condition. The same method is also used for outward solidification. Pedroso and Domoto [4] applied the method of strained coordinates to obtain a perturbation solution for inward solidification of a saturated liquid in spherical coordinates. The temperature at the boundary is assumed constant, but it is shown that the same technique is applicable to other types of boundary conditions. Riley et al. [5] presented an analytical study of the freezing of a liquid inside a sphere or a cylinder which is initially at the fusion temperature, when the outside surface temperature is suddenly lowered to a value less than the fusion one. The perturbation method is used in the solution and the ratio of the latent heat to the sensible heat is assumed to be large. Solomon [6] presented an analytical expression for the total melting time of a slab, a cylinder and a sphere, initially at fusion temperature and subject to convective boundary condition. Voller and Cross [7] developed an explicit algorithm in order to obtain the solidification and melting time of circular regions with first kind of boundary condition. They derived a non-dimensional expression to predict the solidification/melting time of a circular cylinder. They also used this expression to determine upper and lower bounds of solidification/melting time of symmetric cylindrically shaped regions. Hill and Kucera [8] developed a semi-analytical method for the problem of freezing a saturated liquid inside 
a spherical container. They included the effect of radiation at the container surface beside convection. They gave estimates of the time for complete solidification of the sphere. Milanez [9] compared the exact solutions for the slab with numerical solutions obtained for the sphere under the same initial and boundary conditions and developed a correction factor which when applied to the exact solution of the semi-infinite slab will give a solution valid for the sphere. Prud'homme et al. [10] applied the method of strained coordinates to study the inward solidification in slabs, cylinders and spheres for three different types of boundary conditions. It is assumed that the liquid is initially at the fusion temperature and the thermal properties are constant throughout the process. Caldwell and Chan [11] applied a numerical scheme based on the enthalpy method to spherical solidification. The material inside the sphere is assumed at fusion temperature initially and constant temperature boundary condition is applied on the surface. The results are compared to the ones obtained by heat balance integral method. Ismail and Henriquez [12] presented a numerical study of the solidification of a material enclosed in a spherical container. The finite difference approximation and moving grid approach is used throughout the procedure. Constant temperature and convective heat transfer boundary conditions are taken into account. The effects of the size, thickness and material of the container and the external wall temperature on the solidification rate are analysed. Ismail et al. [13] reported the results of a numerical study for the solidification of water in spherical container under convective boundary conditions. The size and the material of the container, external temperature, initial temperature of water are investigated and their effects on the solidification rate and complete solidification time are discussed.

This paper reports the results of a numerical study on the inward solidification of a liquid PCM encapsulated in a cylindrical/spherical container with an initial temperature different than the fusion value. Third kind of boundary condition is considered at the container surface. The enthalpy method with control volume approach is used in the formulation. The governing dimensionless equations are solved many times to obtain data sets for different values of the affecting dimensionless parameters, namely, Stefan Number, Biot Number and Superheat Parameter. These data then correlated to give total solidification time in terms of these parameters.

\section{Mathematical formulation}

Consider a cylindrical/spherical container filled with a liquid PCM at an initial temperature $T_{\text {initial, }}$, which is higher than its fusion temperature $T_{\mathrm{m}}$. At $t=0$, the external boundary of the container is exposed to a fluid with a temperature of $T_{\infty}$, which is lower than $T_{\mathrm{m}}$. Due to the temperature difference, heat exchange takes place between the fluid and PCM by convection. PCM starts to solidify from the outer boundary of the container after the temperature at that boundary reaches $T_{\mathrm{m}}$ and phase change front moves toward the center. The time at which phase change front reaches the center of the container is defined as the total solidification time of the PCM.

The following assumptions are considered in the formulation:

(a) The container wall is so thin and its material is so conductive that the thermal resistance through the wall is negligible.

(b) The temperature of the coolant fluid $T_{\infty}$ and the convective heat transfer coefficient $\mathrm{h}$ are constant. 
(c) The heat transfer process inside the container is only by conduction in radial direction.

(d) The densities of solid and liquid phases of PCM are equal.

Under these assumptions the governing dimensionless enthalpy equation and initial and boundary equations become,

$$
\frac{\partial H^{*}}{\partial \tau}=\frac{1}{R^{n}} \frac{\partial}{\partial R}\left(K^{*} R^{n} \frac{\partial \theta}{\partial R}\right)
$$

where, $n= \begin{cases}1 & \text { for cylindrical container } \\ 2 & \text { for spherical container }\end{cases}$

Initial condition : $\theta(R, 0)=1$

Boundary condition : $K^{*} \frac{\partial \theta}{\partial R}=-B i(\theta)_{R=1}$

The dimensionless enthalpy is defined as

$$
H^{*}(\theta)= \begin{cases}\theta-\theta_{\mathrm{m}} & \text { for } \theta<\theta_{\mathrm{m}} \\ C^{*}\left(\theta-\theta_{\mathrm{m}}\right)+\frac{1}{\text { Ste }} & \text { for } \theta>\theta_{\mathrm{m}}\end{cases}
$$

The enthalpy temperature relation is as follows:

$$
\theta= \begin{cases}H^{*}+\theta_{\mathrm{m}} & \text { for } H^{*}<0 \\ \theta_{\mathrm{m}} & \text { for } 0 \leqslant H^{*} \leqslant \frac{1}{\text { Ste }} \\ \frac{H^{*}-\frac{1}{S t e}}{C^{*}}+\theta_{\mathrm{m}} & \text { for } H^{*}>\frac{1}{\text { Ste }}\end{cases}
$$

The cylindrical/spherical solution region can be divided into small control volumes to apply numerical formulation (Fig. 1). The interfaces of the control volumes are placed at the midway between the nodes. If the control volume approach is applied to the dimensionless enthalpy

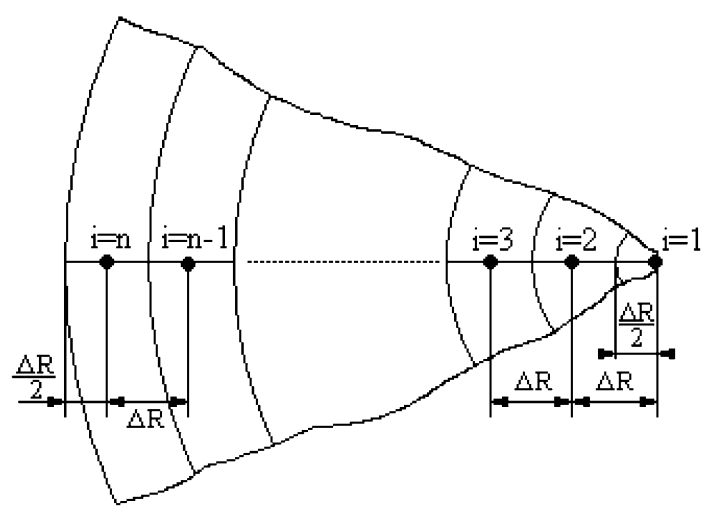

Fig. 1. Control volumes and nodal points inside the cylindrical/spherical container. 
equation (Eq. (1)) and explicit scheme is used then the following equations for different nodes are obtained.

For $i=1$

$$
H_{1}^{*^{k+1}}=H_{1}^{*^{k}}+\frac{m \cdot \Delta \tau}{(\Delta R)^{2}}\left(K_{1}^{*}+K_{2}^{*}\right)\left(\theta_{2}^{k}-\theta_{1}^{k}\right)
$$

where, $m= \begin{cases}2 & \text { for cylindrical container } \\ 3 & \text { for spherical container }\end{cases}$

When deriving Eq. (6), the thermal conductivity at the interface $w$ is taken as the arithmetic mean of the conductivities of neighbour nodes $(W, P)$ (Fig. 2a).

For $i=2,3, \ldots, n-1$

$$
\begin{aligned}
& H_{\mathrm{P}}^{*^{k+1}}=H_{\mathrm{P}}^{*^{k}}+\frac{1}{R_{\mathrm{P}}} \frac{\left[\left(K_{\mathrm{w}}^{*} R_{\mathrm{w}} \frac{\theta_{\mathrm{W}}^{k}-\theta_{\mathrm{P}}^{k}}{\Delta R}\right)-\left(K_{\mathrm{e}}^{*} R_{\mathrm{e}} \frac{\theta_{\mathrm{P}}^{k}-\theta_{\mathrm{E}}^{k}}{\Delta R}\right)\right]}{\Delta R} \Delta \tau \text { for cylinder } \\
& H_{\mathrm{P}}^{*^{k+1}}=H_{\mathrm{P}}^{*^{k}}+\frac{3 \Delta \tau}{\left(R_{\mathrm{w}}^{2}+R_{\mathrm{w}} R_{\mathrm{e}}+R_{\mathrm{e}}^{2}\right)} \frac{\left[\left(K_{\mathrm{w}}^{*} R_{\mathrm{w}}^{2} \frac{\theta_{\mathrm{W}}^{k}-\theta_{\mathrm{P}}^{k}}{\Delta R}\right)-\left(K_{\mathrm{e}}^{*} R_{\mathrm{e}}^{2} \frac{\theta_{\mathrm{P}}^{k}-\theta_{\mathrm{E}}^{k}}{\Delta R}\right)\right]}{\Delta R} \text { for sphere }
\end{aligned}
$$

where $K_{\mathrm{w}}^{*}$ and $K_{e}^{*}$ are the thermal conductivities at the interfaces w and e, respectively (Figs. $2 \mathrm{~b}$ and $2 \mathrm{c}$ ) and are the harmonic means of the conductivities of neighbour nodes.

- for cylinder

$$
\begin{aligned}
& K_{\mathrm{e}}^{*}=\frac{K_{\mathrm{P}}^{*} \cdot K_{\mathrm{E}}^{*} \cdot \ln \left(\frac{i-1}{i-2}\right)}{K_{\mathrm{E}}^{*} \cdot \ln \left(\frac{i-1}{i-1.5}\right)+K_{\mathrm{P}}^{*} \cdot \ln \left(\frac{i-1.5}{i-2}\right)} \\
& K_{\mathrm{w}}^{*}=\frac{K_{\mathrm{W}}^{*} \cdot K_{\mathrm{P}}^{*} \cdot \ln \left(\frac{i}{i-1}\right)}{K_{\mathrm{P}}^{*} \cdot \ln \left(\frac{i}{i-0.5}\right)+K_{\mathrm{W}}^{*} \cdot \ln \left(\frac{i-0.5}{i-1}\right)}
\end{aligned}
$$

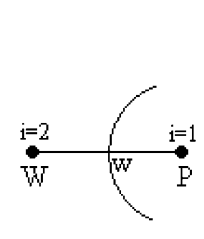

(a)

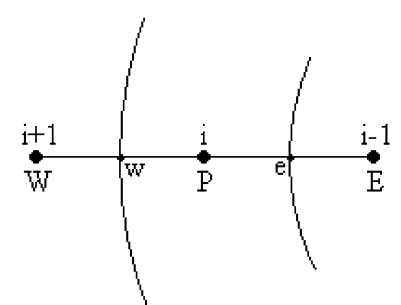

(b)

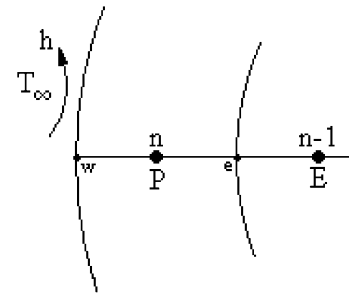

(c)

Fig. 2. (a) First, (b) internal, (c) last control volumes of the cylindrical/spherical container. 
- for sphere

$$
\begin{aligned}
K_{\mathrm{e}}^{*} & =\frac{(i-1.5) K_{\mathrm{E}}^{*} K_{\mathrm{P}}^{*}}{0.5(i-1) K_{\mathrm{P}}^{*}+0.5(i-2) K_{\mathrm{E}}^{*}} \\
K_{\mathrm{w}}^{*} & =\frac{(i-0.5) K_{\mathrm{P}}^{*} K_{\mathrm{W}}^{*}}{0.5 i \cdot K_{\mathrm{W}}^{*}+0.5(i-1) K_{\mathrm{P}}^{*}}
\end{aligned}
$$

For $i=n$

$$
\begin{aligned}
H_{n}^{*^{k+1}}= & H_{n}^{*^{k}}+\frac{\Delta \tau}{(n-1)(\Delta R)^{2}} \\
& \times\left[-\frac{2 B i K_{n}^{*}}{2 K_{n}^{*}+B i \Delta R} \theta_{n}^{k}-\frac{(n-1.5) K_{n}^{*} K_{n-1}^{*} \ln (n-1 / n-2)}{K_{n-1}^{*} \ln (n-1 / n-1.5)+K_{n}^{*} \ln (n-1.5 / n-2)}\left(\theta_{n}^{k}-\theta_{n-1}^{k}\right)\right] \\
& \text { for cylinder } \\
H_{n}^{*^{k+1}}= & H_{n}^{*^{k}}+\frac{3 \Delta \tau}{\left(\Delta R+(n-1.5)(\Delta R)^{2}+(n-1.5)^{2}(\Delta R)^{3}\right)} \\
& \times\left[-\frac{2 B i K_{n}^{*}}{2 K_{n}^{*}+B i \Delta R} \theta_{n}^{k}-\frac{(n-1.5)^{3} K_{n-1}^{*} K_{n}^{*}}{0.5(n-1) K_{n}^{*}+0.5(n-2) K_{n-1}^{*}} \Delta R\left(\theta_{n}^{k}-\theta_{n-1}^{k}\right)\right] \text { for sphere }
\end{aligned}
$$

The dimensionless total heat in the control volume $\mathrm{e}_{i}$ is defined as $H_{i}^{*} V_{e_{i}}^{*}$ where $V_{e_{i}}^{*}$ is the dimensionless volume of the control volume;

$$
H_{i}^{*} V_{e_{i}}^{*}=\left[\left(\theta_{i}-\theta_{\mathrm{m}}\right) V_{\mathrm{s}}^{*}+\left(C^{*}\left(\theta_{i}-\theta_{\mathrm{m}}\right)+\frac{1}{S t e}\right) V_{1}^{*}\right]
$$

This equation can also be expressed as;

$$
H_{i}^{*} \pi\left(R_{\mathrm{w}}^{2}-R_{\mathrm{e}}^{2}\right)=\left[\left(\theta_{i}-\theta_{\mathrm{m}}\right) \pi\left(R_{\mathrm{w}}^{2}-R_{\mathrm{s}}^{2}\right)+\left(C^{*}\left(\theta_{i}-\theta_{\mathrm{m}}\right)+\frac{1}{S t e}\right) \pi\left(R_{\mathrm{s}}^{2}-R_{\mathrm{e}}^{2}\right)\right]
$$

for cylindrical geometry, and

$$
H_{i}^{*} \frac{4}{3} \pi\left(R_{\mathrm{w}}^{3}-R_{\mathrm{e}}^{3}\right)=\left[\left(\theta_{i}-\theta_{\mathrm{m}}\right) \frac{4}{3} \pi\left(R_{\mathrm{w}}^{3}-R_{\mathrm{s}}^{3}\right)+\left(C^{*}\left(\theta_{i}-\theta_{\mathrm{m}}\right)+\frac{1}{S t e}\right) \frac{4}{3} \pi\left(R_{\mathrm{s}}^{3}-R_{\mathrm{e}}^{3}\right)\right]
$$

for spherical geometry.

When freezing front is on the node $i, R_{\mathrm{s}}=\frac{R_{\mathrm{w}}+R_{\mathrm{e}}}{2}$ and $\theta_{i}=\theta_{\mathrm{m}}$, so the dimensionless enthalpy value at this time is;

$$
H_{i}^{*}=\frac{1}{4 S t e} \frac{R_{\mathrm{w}}^{2}+2 R_{\mathrm{w}} R_{\mathrm{e}}-3 R_{\mathrm{e}}^{2}}{R_{\mathrm{w}}^{2}-R_{\mathrm{e}}^{2}}
$$


for cylindrical geometry, and

$$
H_{i}^{*}=\frac{1}{8 S t e} \frac{R_{\mathrm{w}}^{3}+3 R_{\mathrm{w}}^{2} R_{\mathrm{e}}+3 R_{\mathrm{w}} R_{\mathrm{e}}^{2}-7 R_{\mathrm{e}}^{3}}{R_{\mathrm{w}}^{3}-R_{\mathrm{e}}^{3}}
$$

for spherical geometry.

So, when $H_{i}^{*^{k+1}}$ is smaller and $H_{i}^{*^{k}}$ is greater than the quantities on the right sides of the Eqs. (13a) and (13b) for cylindrical and spherical geometries, respectively, this means that the phase change front has just passed through the node i. Furthermore, if it is assumed that the enthalpy changes linearly in any time interval, then the time at which the phase change front is on the node i can be found by;

$$
\tau_{i}=(k+X) \delta \tau
$$

where, $X$ is estimated via linear interpolation in time domain (Fig. 3).

$$
X=\frac{A-H_{i}^{*^{k}}}{H_{i}^{*^{k}}-H_{i}^{*^{k+1}}}
$$

as the result,

$$
X=\frac{\frac{1}{4 S t e} \frac{R_{\mathrm{w}}^{2}+2 R_{\mathrm{w}} R_{\mathrm{e}}-3 R_{\mathrm{e}}^{2}}{R_{\mathrm{w}}^{2}-R_{\mathrm{e}}^{2}}-H_{i}^{*^{k}}}{H_{i}^{*^{k}}-H_{i}^{*^{k+1}}}
$$

for cylindrical geometry, and

$$
X=\frac{\frac{1}{8 S t e} \frac{R_{\mathrm{w}}^{3}+3 R_{\mathrm{w}}^{2} R_{\mathrm{e}}+3 R_{\mathrm{w}} R_{\mathrm{e}}^{2}-7 R_{\mathrm{e}}^{3}}{R_{\mathrm{w}}^{3}-R_{\mathrm{e}}^{3}}-H_{i}^{*^{k}}}{H_{i}^{*^{k}}-H_{i}^{*^{k+1}}}
$$

for spherical geometry.

The following algorithm summarizes the method used to find the dimensionless total solidification time of a cylindrical/spherical container;

(1) Dimensionless enthalpy values of all nodes are calculated at every time step from Eqs. (6), (7a) and (10a) for cylindrical, from Eqs. (6), (7b) and (10b) for spherical container.

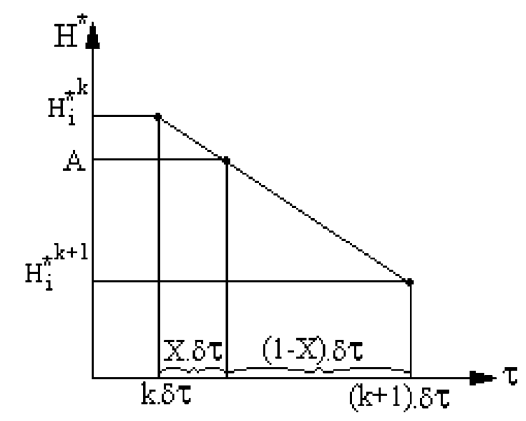

Fig. 3. Dimensionless enthalpy values at the time interval between $k$ and $k+1$. 
(2) The position of the phase change front is determined by comparing the dimensionless enthalpy values of all nodes with the enthalpy value given by Eq. (13a) for cylindrical and Eq. (13b) for spherical container.

(3) The time at which the freezing front reaches the node is calculated by using Eqs. (14) and (15a) for cylindrical and Eqs. (14) and (15b) for spherical container. When the freezing front reaches the first node, which is located at the center of the container, the dimensionless total solidification time of the PCM inside the container is found.

\section{Verification of the method}

In order to validate the numerical code written for cylindrical and spherical containers, comparisons are made with the results of some studies available in the literature.

The dimensionless total solidification times for different Stefan and Biot numbers obtained from this study are compared with the results of [1] in Figs. 4 and 5, for cylindrical and spherical geometries, respectively, in which the liquid PCM is initially at the fusion temperature.

The results of present study are also compared with the results of another work [7], in which the liquid is initially superheated (Fig. 6). Actually, the methods used in both studies are the same and therefore a very good agreement is obtained. The results of [7] are valid for a cylinder with constant temperature boundary condition at the surface, therefore a very big Biot number is chosen during the analysis to make the comparison possible.

The results are also compared with of [13], which investigates the solidification of water in spherical container under convective boundary condition (Fig. 7). The results are generally in



Fig. 4. Comparison of the total solidification time of a cylinder with Ref. [1]. 


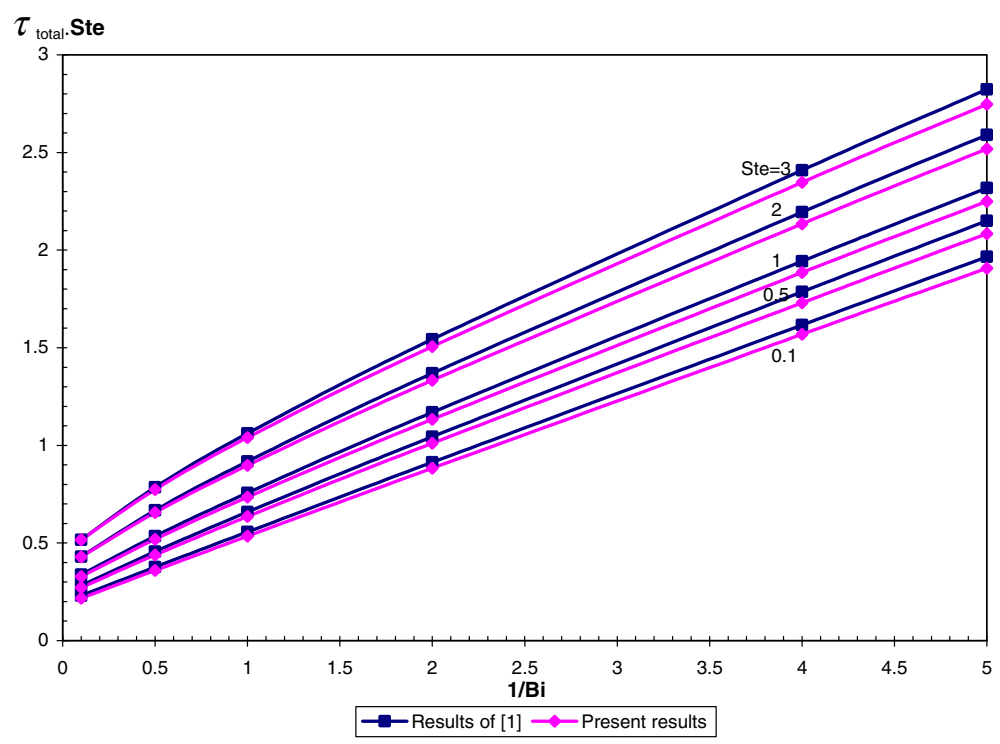

Fig. 5. Comparison of the total solidification time of a sphere with Ref. [1].

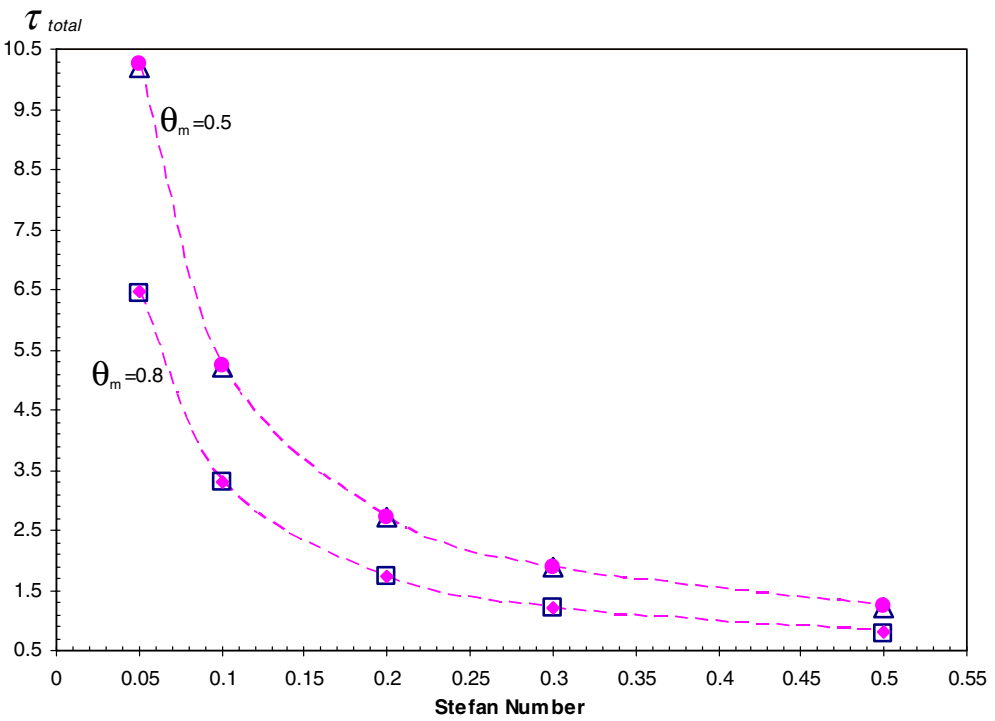

口 $\quad$ Results of $[7]-\bullet--$ Present Results $\quad \Delta \quad$ Results of $[7]----$ Present Results

Fig. 6. Comparison of the total solidification time of a cylinder with Ref. [7].

good agreement. The small differences are due to the exception of the thermal resistance of the container and the time dependency of the convection coefficient in the present study. It can be observed from Fig. 7 that while the results of both studies are very close for smaller containers, as the diameter of the container increases, the discrepancy between the results is getting larger. 


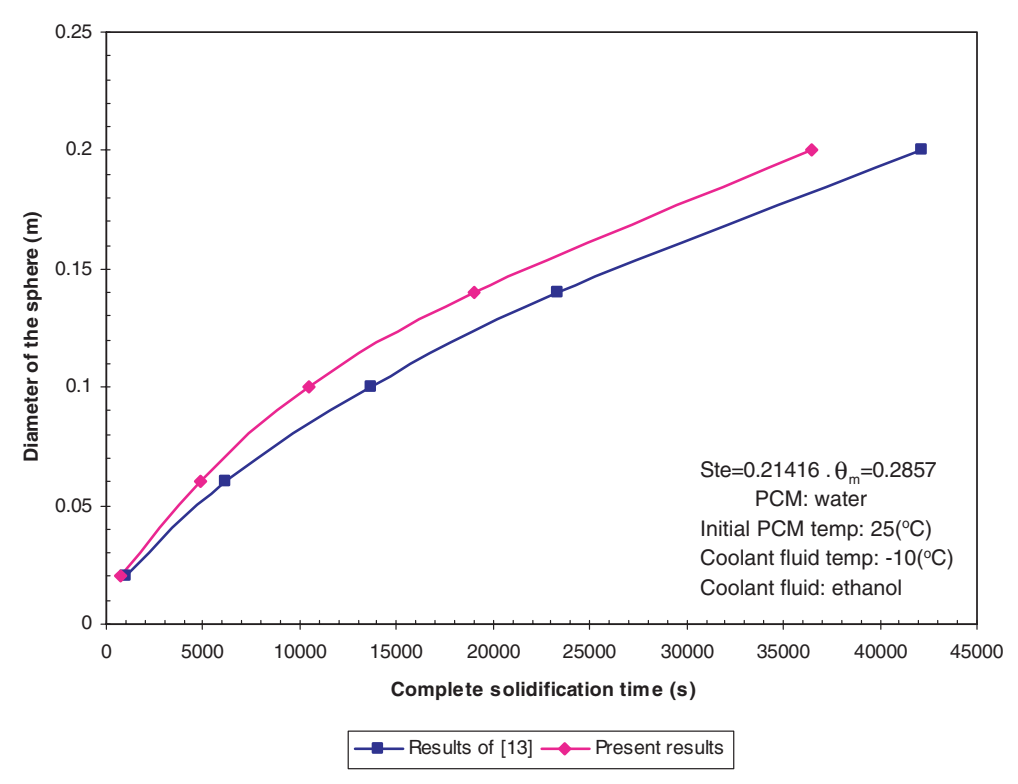

Fig. 7. Comparison of the total solidification time of a sphere with Ref. [13].

The reason of this increase in the difference is the constant convection coefficient assumption made in the present study. When the spherical container diameter is small, this assumption does not lead to a great difference in total solidification time compared to the case of great sphere diameter in which the difference is larger. When the container is small, it does not take much time to solidify completely, so the change in convection coefficient depending on the surface temperature of the container does not affect the results too much. But the situation is different for a big container; in that case solidification takes a longer time and therefore, the difference between two cases becomes larger.

Some oscillations in nodal temperature values are encountered during the solidification process. The reason of these numerical oscillations is the use of harmonic mean approximation for the calculation of thermal conductivity values at the interfaces of control volumes. Voller and Swaminathan [14] also indicated that temperature predictions obtained with the harmonic and arithmetic mean approximations quite often show such oscillations. However, when the total grid number inside the container $(N)$ is increased, it is observed that there is a reduction in numerical fluctuations in temperature values. This effect can be seen from Fig. 8 which shows the change of surface temperature of a spherical container with time. In addition, it is found that the numerical oscillations are not affected when smaller time step values are used.

Since explicit finite difference technique is used in numerical calculations, it is needed to perform a stability analysis. To assure numerical stability, the coefficient of the dimensionless temperature value of the investigated node at the $k^{\text {th }}$ time level $\left(\theta_{i}^{k}\right)$ should be equal to or greater than 0 .

If the first node of a spherical container is considered as an example, the numerical equation for this node can be written as follows: 


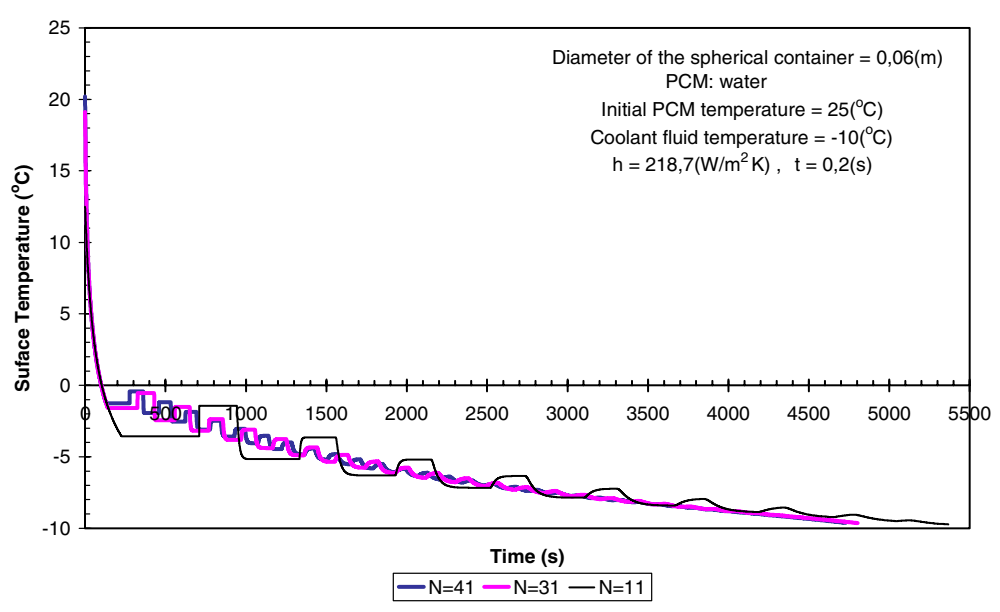

Fig. 8. Surface temperature of a sphere for different total grid numbers.

$$
H_{1}^{*^{k+1}}=H_{1}^{*^{k}}+\frac{3 \Delta \tau}{(\Delta R)^{2}}\left(K_{1}^{*}+K_{2}^{*}\right)\left(\theta_{2}^{k}-\theta_{1}^{k}\right)
$$

Here the dimensionless enthalpy value of the first node at time level $k\left(H_{1}^{*^{k}}\right)$ can be written as

$$
H_{1}^{*^{k}}= \begin{cases}\theta_{1}^{k}-\theta_{\mathrm{m}} & \text { for } \theta_{1}<\theta_{\mathrm{m}} \text { (solid) } \\ C^{*}\left(\theta_{1}^{k}-\theta_{\mathrm{m}}\right)+\frac{1}{\text { Ste }} & \text { for } \theta_{1}>\theta_{\mathrm{m}} \text { (liquid) }\end{cases}
$$

The first node is always liquid, because when the phase change front reaches the first node, the program is ended. So, by substituting the dimensionless enthalpy value taken from Eq. (17) into Eq. (16), the right hand side of Eq. (16) becomes:

$$
C^{*}\left(\theta_{1}^{k}-\theta_{\mathrm{m}}\right)+\frac{1}{\text { Ste }}+\frac{3 \Delta \tau}{(\Delta R)^{2}}\left(K_{1}^{*}+K_{2}^{*}\right)\left(\theta_{2}^{k}-\theta_{1}^{k}\right)
$$

The coefficient of the dimensionless temperature value of the first node is obtained from Eq. (18) as;

$$
C^{*}-\frac{3 \Delta \tau}{(\Delta R)^{2}}\left(K_{1}^{*}+K_{2}^{*}\right)
$$

This value should be equal to or greater than 0 to satisfy the stability. As a result, the upper limit for dimensionless time step can be calculated from Eq. (20):

$$
\Delta \tau \leqslant \frac{C^{*}(\Delta R)^{2}}{3\left(K_{1}^{*}+K_{2}^{*}\right)}
$$

In the present study, two cases are investigated. In the first case, water is selected as PCM $\left(C^{*}=\right.$ $\left.C^{+}=2.0637, K^{+}=0.3016\right)$, while in the second case the specific heat and thermal conductivity values for liquid and solid phases of PCM are taken equal $\left(C^{*}=C^{+}=1, K^{*}=K^{+}=1\right)$. To calculate 
the dimensionless time step value to be used in the numerical calculations, the second case should be taken into consideration, because in that case a smaller $\Delta \tau$ value should be used.

A total of 41 nodal points are used for all cases in the study. The corresponding dimensionless radial distance between grid points $(\Delta R)$ is calculated as 0.0246 .

As a result, the upper limit of the dimensionless time step for the first node is determined from Eq. (20) as 0.00010086 .

Similar investigations are made for the inner and last nodes by taking into consideration the $K^{*}$ and $C^{*}$ values which give minimum value for $\Delta \tau$. It is found that $\Delta \tau$ should be smaller than 0.000290714 for the inner nodes and 0.000344818 for the last node of a spherical container.

The result of similar calculations made for a cylindrical container shows that $\Delta \tau$ should be smaller than 0.00015129 for the first node, 0.00030258 for the inner nodes and 0.000343549 for the last node.

Finally, it is decided to use the value of 0.0001 as the dimensionless time step $(\Delta \tau)$ for all cases, because this value satisfies the stability criterion of all cases.

\section{Results and conclusion}

A phase change problem is expressed by heat conduction equations for liquid and solid phases and energy balance equation for the solid-liquid interface. If these equations are written in dimensionless form, it can be seen that dimensionless phase change front and as the result, the dimensionless total solidification time of a PCM held in a cylindrical or spherical container has a functional relation expressed as below;

$$
\tau_{\text {total }}=f\left(\text { Bi, Ste }, \theta_{\mathrm{m}}, K^{+}, C^{+}\right)
$$

In this study, it is aimed to find correlations giving the dimensionless total solidification time in terms of affecting parameters indicated in Eq. (21).

The correlations are obtained for the case of equal specific heat and thermal conductivity values for liquid and solid phases $\left(K^{+}=1, C^{+}=1\right)$ and for water, which is one of the most common phase change material $\left(K^{+}=0.3016, C^{+}=2.0637\right)$.

The correlations to be found are in the form of,

$$
\tau_{\text {total }}=a \times(\text { Ste })^{b} \times(B i)^{c} \times\left(\theta_{\mathrm{m}}\right)^{d}
$$

To find the coefficients a,b,c and d, the codes written for cylindrical and spherical containers are run for many times for different values of Stefan Number, Biot Number and Superheat Parameter values and data sets are obtained for the dimensionless total solidification time depending on these parameters, then the correlations in the form given by Eq. (22) are obtained by applying multiple regression analysis to these data.

The values of the parameters while the data sets are created are;

$$
\theta_{\mathrm{m}}=\underbrace{0.2,0.3,0.4,0.5,0.6,0.7,0.8,0.9 \text { and } 1}_{9 \text { values }}
$$




$$
\begin{aligned}
& \text { Ste }=\underbrace{0.01,0.025,0.05,0.075,0.1,0.125,0.15, \ldots, 0.45,0.475 \text { and } 0.5}_{21 \text { values }} \\
& B i=\underbrace{1,2,3,4,5, \ldots, 47,48,49 \text { and } 50}_{50 \text { values }}
\end{aligned}
$$

So, a total of 9450 values are calculated for the dimensionless total solidification time, and then the data is correlated to give the following correlations:

(a) For the case of equal thermal conductivity and specific heat values of solid and liquid phases of PCM

(i) For a cylindrical container

$$
\tau_{\text {total }}=0.6496729 \times(\text { Ste })^{-0.9439889} \times(B i)^{-0.194324} \times\left(\theta_{\mathrm{m}}\right)^{-0.9548947}
$$

(ii) For a spherical container

$$
\tau_{\text {total }}=0.4527292 \times(\text { Ste })^{-0.9355889} \times(B i)^{-0.194888} \times\left(\theta_{\mathrm{m}}\right)^{-0.9368148}
$$

(b) For water

(i) For a cylindrical container

$$
\tau_{\text {total }}=0.7192535 \times(\text { Ste })^{-0.9107597} \times(B i)^{-0.188035} \times\left(\theta_{\mathrm{m}}\right)^{-1.010093}
$$

(ii) For a spherical container

$$
\tau_{\text {total }}=0.5012181 \times(\text { Ste })^{-0.9070384} \times(B i)^{-0.1864788} \times\left(\theta_{\mathrm{m}}\right)^{-0.9843633}
$$

The correlation coefficients for each of the correlations are higher than 0.996, which means that these correlations found as the result of multiple regression analysis represent the dimensionless total solidification time found from the solutions very well and can be used in engineering problems with high reliability.

\section{References}

[1] L.C. Tao, Generalized numerical solutions of freezing a saturated liquid in cylinders and spheres, AIChE Journal 13 (1) (1967) 165-169.

[2] S.H. Cho, J.E. Sunderland, Phase change of spherical bodies, International Journal of Heat and Mass Transfer 13 (1970) 1231-1233.

[3] Y.P. Shih, S.Y. Tsay, Analytical solutions for freezing a saturated liquid inside and outside cylinders, Chemical Engineering Science 26 (1971) 809-816.

[4] R.I. Pedroso, G.A. Domoto, Inward spherical solidification—solution by the method of strained coordinates, International Journal of Heat and Mass Transfer 16 (1973) 1037-1043.

[5] D.S. Riley, F.T. Smith, G. Poots, The inward solidification of spheres and circular cylinders, International Journal of Heat and Mass Transfer 17 (1974) 1507-1516.

[6] A.D. Solomon, On the melting time of a simple body with a convection boundary condition, Letters in Heat and Mass Transfer 7 (1980) 183-188. 
[7] V.R. Voller, M. Cross, Estimating the solidification/melting times of cylindrically symmetric regions, International Journal of Heat and Mass Transfer 24 (9) (1981) 1457-1462.

[8] J.M. Hill, A. Kucera, Freezing a saturated liquid inside a sphere, International Journal of Heat and Mass Transfer 26 (11) (1983) 1631-1637.

[9] L.F. Milanez, Simplified relations for the phase change process in spherical geometry, International Journal of Heat and Mass Transfer 28 (4) (1985) 884-885.

[10] M. Prud'homme, T.H. Nguyen, D.L. Nguyen, A heat transfer analysis for solidification of slabs, cylinders and spheres, Journal of Heat Transfer 111 (1989) 699-705.

[11] J.D. Caldwell, C.C. Chan, Spherical solidification by the enthalpy method and heat balance integral method, Applied Mathematical Modelling 24 (2000) 45-53.

[12] K.A.R. Ismail, J.R. Henriquez, Solidification of PCM inside a spherical capsule, Energy Conversion and Management 41 (2000) 173-187.

[13] K.A.R. Ismail, J.R. Henriquez, T.M. da Silva, A parametric study on ice formation inside a spherical capsule, International Journal of Thermal Sciences 42 (2003) 881-887.

[14] V.R. Voller, C.R. Swaminathan, Treatment of discontinuous thermal conductivity in control volume solutions of phase change problems, Numerical Heat Transfer Part B 24 (1993) 161-180.

Levent BİLIR was awarded a B.Sc. degree at Dokuz Eylül University, İzmir, Turkey, and a M.Sc. degree at İzmir Institute of Technology, Turkey. He is currently a Ph.D student and research assistant in mechanical engineering department at İzmir Institute of Technology.

Zafer ILKEN gained his B.Sc. at Middle East Technical University, Ankara, Turkey, M.Sc. and Ph.D. degrees from Dokuz Eylül University, İzmir, Turkey. He is employed as a full professor at İzmir Institute of Technology in 1999. His research areas are thermal energy storage and renewable energy sources. 